\title{
Tumour-to-tumour Metastasis: Cutaneous Melanoma Metastasizing to Leiomyoma Uteri
}

\author{
Jelena Amidzic1, Nenad Solajic², Aleksandra Fejsa Levakov1, Matilda Djolai1 and Nada Vuckovic² \\ 1Department for Histology and Embryology / Pathology2, Centre for Pathology and Histology, Clinical Centre of Vojvodina, \\ Faculty of Medicine, University of Novi Sad, HajdukVeljkova 1-3, 21000 Novi Sad, Serbia
}

\begin{abstract}
The incidence of melanoma is increasing worldwide. It is known that melanoma frequently progresses to metastatic disease.

The aim of this report is to emphasise the metastatic potential of cutaneous melanoma to various body areas, as well as the ability to produce unexpected presentation of the disease.

A 48-year female had a myomatous uterus and underwent hysterectomy. At the pathological examination, multiple leiomyomas were diagnosed and in one of them, the metastatic melanoma was found, the later confirmed with immunohistochemical analysis. The medical history revealed that the patient was previously operated two years back due to skin superficial spreading melanoma.

The metastasis to uterine leiomyoma was the first site of distant spread.

Melanoma is a type of tumour with aggressive and unpredictable behaviour, so metastases to unexpected localisations could occur. A careful examination of patient's body is mandatory, including the remote areas and even benign tumours.
\end{abstract}

Key Words: Tumout-to-tumour metastasis, Melanoma, Leiomyoma.

How to cite this article: Amidzic J, Solajic N, Fejsa Levakov A, Djolai M, Vuckovic N. Tumour-to-tumour metastasis: cutaneous melanoma metastasizing to leiomyoma uteri. J Coll Physicians Surg Pak 2019; 29 (Supplement 2):S112-S113.

\section{INTRODUCTION}

Melanoma is an aggressive, highly malignant disease that is derived from melanocytes. The incidence of melanoma is increasing worldwide. In more than $90 \%$ of cases, the primary localisation of such tumours is in the skin. Less often, melanoma may primarily occur in the eye, leptomeninges and mucous membranes of respiratory, digestive, and genitourinary tracts. The primary localisation of melanoma is unknown in about $2 \%$ of reported cases. Melanoma has a strong tendency for metastasis. After primary excision of tumour, about $30 \%$ of all patients develop distant metastases within first 5 years after tumour diagnosis. ${ }^{1}$

Differentiating metastatic melanoma from epithelial, mesenchymal and neural tumours by means of routine histology, can be quite challenging since melanomas may have very diverse histological appearances and also may lack visible melanin pigment. Immunohistochemistry is, therefore, often necessary in diagnosing metastatic melanoma. The usual combination of immunohistochemical markers for detecting primary and metastatic melanoma is HMB-45, Melanoma Antigen

Correspondence to: Jelena Amidzic, Department for Histology and Embryology, Faculty of Medicine, University of Novi Sad Centre for Pathology and Histology, Clinical Centre of Vojvodina HajdukVeljkova 1-3, 21000 Novi Sad, Serbia

E-mail: jelena.amidzic@mf.uns.ac.rs

Received: May 06, 2019; Revised: July 10, 2019;

Accepted: July 10, 2019
Recognised by T-cells (MART1) and polyclonal or monoclonal S100 protein. ${ }^{2}$

The aim of this case report is to highlight the widespread metastatic potential of the melanoma as well as its tendency for unusual presentation of metastatic disease.

\section{CASE REPORT}

A 48-year female patient had undergone a hysterectomy because of myomatous uterus. After the surgery, the material was submitted for pathohistological analysis.

Grossly, uterine dimensions were $7 \times 8 \times 6 \mathrm{~cm}$. On crosssections within myometrium, there were 4 round, sharply circumscribed, greyish-white nodules, with a whorled, fibrillar appearance on cut surface, measuring from 0.5 to $1.6 \mathrm{~cm}$ (Figure 1A). The histological examination led to the diagnosis of multiple uterine leiomyomas. In the central part of the biggest, subserous positioned leiomyoma, there was another tumour tissue $0.2 \mathrm{~cm}$ in diameter, with infiltrative margins, composed of smaller groups and pseudo-papillary formations of atypical cells with scant cytoplasm, large round to ovoid nuclei, and prominent nucleoli, without the intracytoplasmic pigment (Figure 1B).

Immunohistochemical staining of the tumour cells showed cytoplasmic positivity of MART-1 (Figure 2A) and cytoplasmic and nuclear positivity of $\mathrm{S} 100$ protein (Figure 2B).

A diagnosis of stage IV melanoma was established (metastasis of melanoma to uterine leiomyoma). 


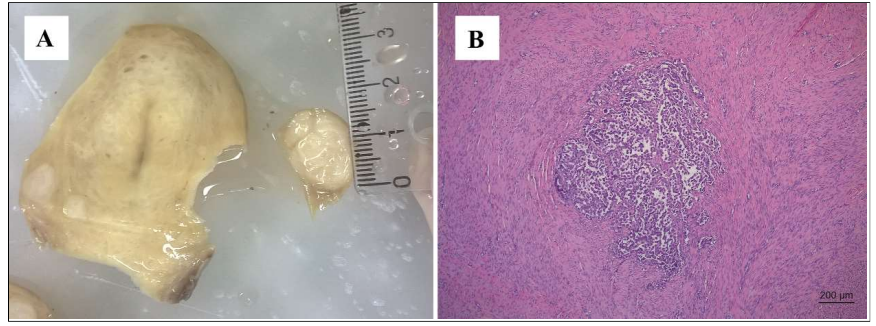

Figure 1: (A) Gross section shows nodule measuring $1.6 \mathrm{~cm}$ in diameter within myometrium. (B) Hematoxylin and eosin stained section ilustrates metastatic melanoma within typical leiomyoma (original magnification 100x).

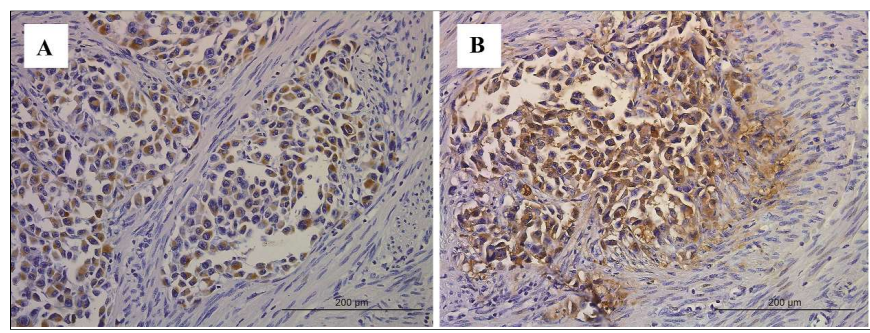

Figure 2: Immunohistochemistry shows positivite staining of the tumour cells and supports the diagnosis of metastatic melanoma;

(A) Immunohistochemical staining for MART1 (original magnification 400x).

(B) Immunohistochemical staining for $\mathrm{s} 100$ protein (original magnification 400x).

Insight into the medical records revealed that the patient underwent a surgery to remove a nevus above her left breast 2 years prior to this hysterectomy. At the time, she was diagnosed with superficially spreading melanoma, with $7 \mathrm{~mm}$ in greatest diameter, and maximum tumour thickness of $2.1 \mathrm{~mm}$ (Clark IV, Breslow III), as well as 2 tumour-involved nodes, from the left axillary region, out of 13 examined (pT3aN2bM0).

In less than a month after the diagnosis of the tumour, metastasis in the uterus, the radiology examination showed dissemination of the disease and multiple metastasis in the adrenal glands, anterior abdominal wall, brain, lungs, abdomen and minor pelvis. The tumour board stated that the patient was inoperable, and after 5 series of vemurafenib therapy, further disease progression was noticed.

\section{DISCUSSION}

The presented case highlights the enormous metastatic potential of cutaneous melanoma.

In 2015, Venyo et al. published a review article on reported cases of melanoma metastatic to uterus and concluded that this phenomenon is extremely rare, and in addition, the reported cases had poor prognosis. By using various internet search engines and biomedical databases, they found 35 reported cases in the period from 1974 to 2013.3 In most reported cases, the uterus was grossly altered. In the present case, the tumour tissue of the melanoma was not visible at macroscopic uterus examination, and that was the first diagnosed metastasis, without the existence of known metastatic deposits on other localisations at that moment.

In our patient's case, the metastatic melanoma to uterine leiomyoma was established 2.5 years after surgery of the primary cutaneous tumour.
Regarding benign uterine tumours harbouring secondary deposits of melanoma, mostly accidentally, very few papers are published discussing the presence of metastatic tumour tissue originating from cutaneous melanoma to leiomyoma uteri ${ }^{4}$, as well as to uterine adenomyoma. ${ }^{5}$

Haematogenous distant metastases of melanoma represent stage IV of the disease, where any further prognosis is unfavourable, with average survival rate below 6 months. 6

Unlike other malignant cutaneous tumours, melanoma is a particularly aggressive disease with unpredictable evolution. Due to the fact that it has high metastatic potential as well as the fact that the incidence of its occurrence is increasing, the occurrence of metastases in unusual and unexpected localisations, as is the distant benign tumour in the present case, shall probably happen more often in future.

Our primary goal is to highlight the fact that pathologists should always consider the possibility of presense of melanoma in tumours, which may appear indolent and grossly benign and be fastidious about sampling and examination of histological specimens.

\section{PATIENT'S CONSENT:}

Patient's consent is obtained for operation and use of any material for scientific studies and publishing the data.

\section{CONFLICT OF INTEREST:}

Authors declared no conflict of interest.

\section{AUTHORS' CONTRIBUTION:}

JA, NS, AFL, MD, NV: Conception or design of the study; acquisition of the data; analysis or interpretation of data; drafting of the manuscript; critical revision of the manuscript for important intellectual content, final approval of the version to be submitted; agreement to be accountable for all aspects of the work.

\section{REFERENCES}

1. Jemal A, Ward EM, Johnson CJ, Cronin KA, Ma J, Ryerson B, et al. Annual report to the nation on the status of cancer, 19752014, featuring survival. J Natl Cancer Inst 2017; $109: 9$.

2. Prieto VG, Shea CR. Immunohistochemistry of melanocytic proliferations. Arch Pathol Lab Med 2011; 135:853-9.

3. Venyo LKG, Fatola CO, Venyo AKG. Melanoma of the uterus: A review of the literature. Int J Women's Heal Reprod Sci 2015; 3:2-12.

4. Chanthasenanont A, Nantakomon T, Kintarak J, Vithisuvanakul N, Pongrojpaw D, Suwannarurk K. Incidental finding of metastatic cutaneous malignant melanoma at uterine leiomyoma: A Thai University Hospital experience: A case report. J Med Assoc Thai 2015; 98(Suppl 3):S126-31.

5. Simeone S, Laterza MM, Scaravilli G, Capuano S, Serao M, Orabona $\mathrm{P}$, et al. Malignant melanoma metastasizing to the uterus in a patient with atypical postmenopause metrorrhagia. A case report. Minerva Ginecol 2009; 61:77-80.

6. Sandru A, Voinea S, Panaitescu E, Blidaru A. Survival rates of patients with metastatic malignant melanoma. J Med Life 2014; 7:572-6.

$$
\text { -.......... }
$$

